\title{
El rol de la felicidad y el optimismo como factor protector de la ansiedad
}

\section{The role of happyness and optimism as a protector factor of anxiety}

Recibido: abril 1 de 2011 |Revisado: septiembre 9 de 2011 | Aceptado: noviembre 9 de 2011

\author{
Paula Pavez \\ LUIS MENA \\ Pablo Vera-Villarroel ${ }^{* *}$ \\ Universidad de Santiago de Chile, Santiago, Chile
}

\section{RESUMEN}

Se evaluaron factores protectores y de riesgo asociados a la ansiedad en 711 personas, quienes respondieron escalas de ansiedad, depresión, felicidad y optimismo, más antecedentes sociodemográficos. Los resultados indicaron que la depresión es un factor de riesgo para la ansiedad estado como rasgo, lo que concuerda con la literatura revisada, pues esta variable ha sido detectada en comorbilidad a la ansiedad, en estudios con personas de distintos grupos etarios. La felicidad se constituyó como factor protector para ambas ansiedades, mientras que el optimismo se evidenció como protector de la ansiedad rasgo. Estos resultados destacan la necesidad de mayor investigación con variables positivas y negativas en conjunto, dada sus potenciales aplicaciones en las intervenciones psicoterapéuticas especializadas para los trastornos psicológicos.

Palabras clave autores

Ansiedad, depresión, felicidad, optimismo.

Palabras clave descriptores

Pruebas psicológicas, factores de riesgo, investigación cuantitativa

\section{A B S T R A C T}

Protection and risk factors associated with anxiety were evaluated in 711 people, who reported different values in scales of anxiety, depression, happiness and optimism, including their socio-demographic background.

Evidence that depression is a risk factor for anxiety state as a feature was found, which is in accordance with previous works, since this variable has been observed in comorbility to anxiety in people belonging to different age groups. It is also observed that happiness constitute a protective factor for both anxieties, while optimism is evinced as a protector of the anxiety feature. These results emphasize the need of further research, using positive and negative features as a whole, given the potential applications specialized in psychotherapeutic interventions for psychological disorders since this type of studies continue to be scarce.

Key words authors

Anxiety, depression, happiness, optimism.

Key words plus

Psichological tests, risk factors, quantitative research. 


\section{Introducción}

La prevalencia de la ansiedad en la población mundial muestra rangos que van desde $2.4 \%$ hasta un $18.2 \%$ en diferentes países de América, Europa y Asia (Kessler \& Üstün, 2004). En Chile, esta patología es uno de los principales trastornos psicológicos por los que consultan las personas en los centros de salud después de la depresión (Vicente, Rioseco, Saldivia, Kohn \& Torres, 2002).

En la actualidad, la Psicología cuenta con amplia evidencia en cuanto a cuáles serían los factores de riesgo asociados a esta sintomatología, así como de estrategias para su intervención (Arcas \& Cano, 1999; Sandín, 1995). Sin embargo, existe aún poca información frente a qué factores ligados a agentes de riesgo puedan proteger su aparición (Fredrickson \& Losada, 2005).

Los elementos que protegen de la aparición de sintomatología y promueven la salud mental y el bienestar, han cursado un acrecentamiento relevante durante las últimas décadas, dando paso al estudio de variables positivas y preventivas en lugar de aspectos puramente nocivos y patológicos (Contreras \& Esguerra, 2006; Gable \& Haidt, 2005; Seligman, Steen, Park \& Peterson, 2005). En este sentido, se ha verificado que la variable optimismo tiene una relación negativa con variables como ansiedad, ira, estrés y depresión (Abdel-Khalek, 2006; Brydon, Walker, Wawrzyniak, Chart \& Steptoc, 2009; Chico \& Ferrando, 2008; De Moor, De Moor, Basen-Engquist, Kundelka, Bevers \& Cohen, 2006; Kivimaki, Elovainio, Singh-Manoux, Vahtera, Helenius \& Pentti, 2005; Lobel, DeVincent, Kaminer \& Meyer, 2000; Puskar, Ren, Bernardo, Haley \& Stark, 2008; Vera, 2006; Vera-Villarroel, Zych, Celis-Atenas, CórdovaRubio \& Buela-Casal, 2011), mientras que en sentido opuesto, presenta una interacción positiva con factores como satisfacción con la vida, autoestima (Vittorio et al., 2009) y felicidad y bienestar (Lyubomirsky, 2008; Vera-Villarroel, Códova-Rubio \& Celis-Atenas, 2009a). Igualmente se ha identificado que las personas con una percepción optimista de su enfermedad, tienen una vida más prolongada, promueven la búsqueda de beneficios en su condición y reportan mejor calidad de vida que aquellos que asumen su condición de manera pesimista (Hart, Vella \& Mohr, 2008; Taylor, Kemeny, Reed, Bower \& Gruenewald, 2000).

De la misma forma, otro de estos agentes que protegerían la aparición de síntomas negativos, correspondería a la variable felicidad. Investigaciones en esta línea muestran que esta variable se asocia con el optimismo, aumento de alegría, satisfacción, nivel de energía, mejora del sistema inmunológico, dedicación al trabajo y salud mental (Lyubomirsky, 2008; Moyano, Flores \& Soromaa, 2011), relaciones positivas con el bienestar (Retana-Franco \& SánchezAragón, 2010; Vázquez, Hervás \& Ho, 2006; Vázquez, Hérvas, Rahona \& Gómez, 2009), esperanza de vida y salud física (Koopmans, Geleijnse, Zitman \& Giltay, 2010; Piqueras, Kuhne, Vera-Villarroel, van Straten \& Cuijpers, 2011) y negativamente con la depresión (Cuadra-Peralta, Veloso-Besio, Ibergaray \& Rocha, 2010; Lyubomirsky, 2008; Moyano \& Ramos, 2007).

Aunque la evidencia presentada respecto de los beneficios que aportan estas variables positivas se aprecia relevante, el estudio de estos factores protectores junto a los de riesgo para la ansiedad es escaso (Mustaca, Kamenetzky \& Vera-Villarroel, 2010), a pesar que investigaciones realizadas indicarían que las personas podrían ser menos vulnerables a las emociones negativas por la experimentación de emociones positivas (Fredrickon \& Tugade, 2003).

De acuerdo a esta situación, parece relevante analizar e identificar factores protectores y de riesgo en conjunto para la ansiedad, a modo de soslayar el permanente interés que se ha tenido en aspectos estrictamente patológicos. Igualmente, este tipo de investigaciones podrían ayudar no sólo a la identificación de agentes protectores en conjunto con los de vulnerabilidad, que además a la comprensión y diferenciación de la ansiedad de patologías con las que se ve estrechamente ligada por su considerable comorbilidad, como es la depresión (Beutel, Bleicher, von Heymann, Tritt \& Hardt, 2011; Cova, Rincón \& Mellipán, 2011; Forand, Gunthert, Cohen, Butler \& Beck, 2010; Vera-Villarroel \& Buela-Casal, 2000; Vera-Villarroel, Buela-Casal, Celis-Atenas, CórdovaRubio, Encina-Olea.\& Spielberger, 2008; Watson, Clark \& Carey, 1998; Watson, Clark \& Stasik, 2011). En este sentido, no son pocos los estudios que establecen que esta alta comorbilidad implicaría no 
solo un aumento del deterioro y adición de síntomas, sino también complicaciones para su tratamiento (Forand et al., 2010; Pimentel \& Cova, 2011).

De esta forma, el objetivo de esta investigación es la identificación de posibles agentes protectores y de riesgo en conjunto para la ansiedad. En este interesa evaluar si la felicidad y el optimismo pueden actuar como factores protectores para la ansiedad.

\section{Método}

\section{Participantes}

La muestra total de este estudio estuvo compuesta por 711 personas de la ciudad de Santiago de Chile, entre los 18 y los 71 años de edad. El proceso de selección se realizó mediante un método no probabilístico intencional (Salkind, 1998), se accedió a los participantes que voluntariamente quisieron colaborar en la investigación.

\section{Diseño}

Se utilizó un diseño transeccional, correlacional, predictivo. Como variable criterio se estableció la ansiedad, mientras que como predictoras se dispuso la felicidad, el optimismo, la depresión y las variables sociodemográficas.

\section{Procedimiento}

Para la recolección de los datos se invitó a las personas voluntarias, a contestar individualmente una serie de cuestionarios. Los participantes firmaron previamente un consentimiento informado. Para la administración de los instrumentos, estos fueron previamente explicados y leídos con cada participante.

\section{Instrumentos}

Se utilizaron los siguientes instrumentos:

\section{Optimismo Disposicional}

Test de Orientación Vital revisado (Scheier, Carver \& Bridges, 1994) en la versión española de
Otero-López, Luengo, Romero, Gómez y Castro (1998). Es un instrumento tipo Likert que evalúa expectativas de resultados favorables en el futuro. El LOT-R presenta adecuadas propiedades psicométricas en poblaciones portuguesas (Laranjeira, 2008) y chilenas (Vera-Villarroel, Córdova-Rubio $\&$ Celis-Atenas, 2009b).

\section{Escala de Felicidad General (Lyubomirsky Ë Lepper, 1999)}

Compuesto por cuatro ítems, con una escala de siete puntos (1 a 7), que mide desde el grado más bajo al más alto de Felicidad. Este instrumento presenta adecuadas propiedades psicométricas para población adolescente, universitaria y población chilena (Vera-Villarroel, Celis-Atenas \& CórdovaRubio, 2011).

Inventario de Depresión de Beck ([BDI], Beck, Rush, Shaw \& Emery, 1983)

Evalúa la gravedad e intensidad de los síntomas cognitivos, afectivos, conductuales y psicofisiológicos de la depresión. El rango de la puntuación que se obtiene es de 0 a 63 puntos. Se observan elevados índices de consistencia interna en la validación española del cuestionario, 0.83 y 0.9 (Melipillán Araneda, Cova Solar, Rincón González \& Valdivia Peralta, 2008; Sanz \& Vázquez, 1998; Vázquez \& Sanz, 1997).

Inventario de Ansiedad Estado - Rasgo (Spielberger et al., 1970).

Esta escala, en su forma Ansiedad Estado (A-E), tiene por objetivo evaluar la ansiedad o condición emocional transitoria de la persona, y en su modo Ansiedad Rasgo (A-R) mide la ansiedad como una condición emocional permanente de tensión (Spielberger et al., 1970). La adaptación en población chilena mostró adecuados indicadores psicométricos. La consistencia interna fue de 0.92 para la escala estado y de 0.87 para la escala rasgo, mientras que la estructura factorial estuvo acorde a lo mostrado por otras investigaciones (Vera-Villarroel, Celis- 
Atenas, Córdova-Rubio, Buela-Casal \& Spielberger, 2007). El punto de corte para categorizar esta variable tanto en la escala estado como rasgo, recayó en el puntaje obtenido que se corresponde con el percentil 70 (Vera-Villarroel et al., 2007).

Finalmente, se controlaron las variables edad, sexo, estado civil, nivel educacional, categoría ocupacional y nivel socioeconómico de los participantes; esto último a través del método Esomar (Adimark, 2000) que clasifica la condición socioeconómica con base en dos variables: el nivel de educación alcanzado por el principal sostenedor del hogar y su categoría ocupacional. Ambas variables se combinan en una matriz de clasificación socioeconómica, generándose seis grupos, a saber: A (Muy Alto), B (Alto), CA (Medio-Alto), CB (Medio), D (Medio-Bajo), E (Bajo).

\section{Análisis de Datos}

En un primer momento se analizaron descriptivamente variables sociodemográficas y luego se compararon en función de la variable criterio (ansiedad) recurriendo a la prueba de contraste de Chi cuadrado. Para los análisis predictivos posteriores, se realizaron análisis de regresión logística binaria con las variables del estudio. Estos análisis se realizaron mediante el programa estadístico SPSS 15.0.

\section{Resultados}

Al caracterizar la muestra, se encontró que esta se concentra en un $67 \%$ entre los 18 y los 24 años de edad, con una media de 26.32 y una desviación estándar de 9.801, siendo la mayoría de sexo femenino (57\%). La mayor parte de estas personas se encuentran solteras $(87 \%)$, con un nivel educacional de enseñanza media completa (32\%), siendo su categoría ocupacional de empleado administrativo $(31.3 \%)$ y un nivel socioeconómico medio (32\%) (Tabla 1).

Frente a la ansiedad, se identificó que los sujetos de 18 a 24 años son los que presentan mayor presencia de esta variable, tanto en su forma estado como rasgo. Igualmente, es el sexo femenino el que se aprecia con mayor sintomatología (Tablas 2 y 3 ).

En cuanto al resto de las variables sociodemográficas estudiadas y la ansiedad, tanto para la
TABLA 1

Características sociodemográficas de la muestra

\begin{tabular}{|c|c|c|}
\hline & $N$ & $\%$ \\
\hline \multicolumn{3}{|l|}{ Edad } \\
\hline 18 a 24 años & 475 & 66.81 \\
\hline 25 a 44 años & 170 & 23.91 \\
\hline 45 a 71 años & 66 & 9.28 \\
\hline \multicolumn{3}{|l|}{ Sexo } \\
\hline Femenino & 407 & 57.2 \\
\hline Masculino & 304 & 42.8 \\
\hline \multicolumn{3}{|l|}{ Estado Civil $(N=657) *$} \\
\hline Soltero & 570 & 86.7 \\
\hline Casado & 87 & 13.2 \\
\hline \multicolumn{3}{|c|}{ Nivel educacional $(\mathrm{N}=520)^{*}$} \\
\hline básica incompleta & 25 & 4.81 \\
\hline básica completa & 29 & 5.58 \\
\hline media incompleta & 75 & 14.42 \\
\hline media completa & 168 & 32.31 \\
\hline universitaria incompleta & 122 & 23.46 \\
\hline universitaria completa & 80 & 15.38 \\
\hline posgrado & 21 & 4.04 \\
\hline \multicolumn{3}{|c|}{ Categoría ocupacional $(N=520) *$} \\
\hline $\begin{array}{l}\text { Trabajos menores oca- } \\
\text { sionales }\end{array}$ & 45 & 8.7 \\
\hline Oficio menor & 50 & 9.6 \\
\hline Obrero calificado & 113 & 22.5 \\
\hline $\begin{array}{l}\text { Empleado administra- } \\
\text { tivo }\end{array}$ & 163 & 31.3 \\
\hline Ejecutivo medio & 112 & 21.5 \\
\hline Alto ejecutivo & 33 & 6.3 \\
\hline \multicolumn{3}{|c|}{ Nivel socioeconómico $(n=657) *$} \\
\hline Bajo & 74 & 11.26 \\
\hline Medio bajo & 104 & 15.83 \\
\hline Medio & 213 & 32.42 \\
\hline Medio alto & 180 & 27.40 \\
\hline Alto & 62 & 9.44 \\
\hline Muy alto & 24 & 3.65 \\
\hline
\end{tabular}

Nota. *Al no disponer de los datos completos de los/as participantes, la muestra en esta variable es menor.

Fuente: elaboración propia.

ansiedad estado como rasgo, son las personas solteras, que tienen un nivel educacional de enseñanza media completa, con una categoría ocupacional de empleado administrativo y poseen un nivel socioeconómico medio, las que presentan una mayor sintomatología ansiosa (Tablas 2 y 3). 


\section{Modelo predictivo para la ansiedad estado}

La evaluación de la capacidad predictiva de las variables de estudio en relación con ansiedad estado, se realizó por medio de regresión logística (Tabla 4). Para comprobar que variables optimismo, felicidad, depresión y las sociodemográficas, se asocien con sintomatología ansiosa, se probó un modelo con estas variables psicológicas de estudio. El modelo resultante fue adecuado para la prueba de Hosmer y Lemeshow $(p=0.76)$, indicando que no hay diferencias estadísticamente significativas entre las clasificaciones observadas y las predichas.

TABLA 2

Características sociodemográficas de la muestra de acuerdo a la presencia o ausencia de ansiedad estado (cálculos realizados con prueba de Chi-Cuadrado)

\begin{tabular}{lccc}
\hline \multicolumn{4}{c}{ Ansiedad Estado } \\
\hline & $\begin{array}{c}\text { Ausencia } \\
(\mathbf{N})\end{array}$ & $\begin{array}{c}\text { Presencia } \\
(\mathbf{N})\end{array}$ & X2 \\
\hline Edad & & & $0.047^{*}$ \\
\hline 18 a 24 años & 362 & 113 & \\
25 a 44 años & 128 & 42 & \\
45 a 71 años & 41 & 25 & \\
Total & 531 & 180 & \\
\hline Sexo & & & 0.118 \\
\hline Masculino & 295 & 112 & \\
Femenino & 236 & 68 & \\
Total & 531 & 180 & \\
\hline Estado civil & & & 0.513 \\
\hline Soltero & 427 & 142 & \\
Casado & 68 & 19 & \\
Total & 495 & 162 & \\
\hline Nivel educacional & & & 0.910 \\
\hline Básica incompleta & 20 & 5 & \\
Básica completa & 23 & 6 & \\
Media incompleta & 55 & 20 & \\
Media completa & 129 & 39 & \\
Universitaria incom- & 90 & 32 & \\
pleta & & & \\
Universitaria completa & 62 & 18 & \\
Posgrado & 14 & 7 & \\
\hline Total & 393 & 127 & \\
\hline & & & \\
\hline
\end{tabular}

Nota. $* \mathrm{p}<0.05$.

Fuente: elaboración propia.
Por su parte, el estadístico $R^{2}$ de Nagelkerke estima un valor de ajuste adecuado (0.55) lo que lleva a aceptar formalmente un buen ajuste del modelo y establece la existencia una correlación fuerte $(r=0.74)$ entre todas las variables predictoras y ansiedad estado.

Asimismo, el modelo resultante evidenció una adecuada especificidad (94.7\%) y sensibilidad (63\%), es decir, que clasifica correctamente a aquellas personas que efectivamente no presentan ansiedad estado, como a aquellas personas que sí tienen el atributo. Igualmente, se observa un adecuado potencial global (86.9\%), es decir que existe buena capacidad predictiva global del modelo.

TABLA 3

Características sociodemográficas de la muestra de acuerdo a la presencia o ausencia de ansiedad rasgo (cálculos realizados con prueba de Chi-Cuadrado)

\begin{tabular}{|c|c|c|c|}
\hline \multicolumn{4}{|c|}{ Ansiedad Rasgo } \\
\hline & $\begin{array}{l}\text { Ausencia } \\
(\mathrm{N})\end{array}$ & $\begin{array}{l}\text { Presencia } \\
(\mathrm{N})\end{array}$ & $x^{2}$ \\
\hline Edad & & & $0.007 * *$ \\
\hline 18 a 24 años & 376 & 99 & \\
\hline 25 a 44 años & 134 & 36 & \\
\hline 45 a 71 años & 41 & 25 & \\
\hline Total & 551 & 160 & \\
\hline Sexo & & & $0.024 *$ \\
\hline Masculino & 303 & 104 & \\
\hline Femenino & 248 & 56 & \\
\hline Total & 551 & 160 & \\
\hline Estado civil & & & 0.454 \\
\hline Soltero & 445 & 125 & \\
\hline Casado & 71 & 16 & \\
\hline Total & 516 & 141 & \\
\hline Nivel educacional & & & 0.705 \\
\hline Básica incompleta & 20 & 5 & \\
\hline Básica completa & 24 & 5 & \\
\hline Media incompleta & 57 & 18 & \\
\hline Media completa & 128 & 40 & \\
\hline $\begin{array}{l}\text { Universitaria incom- } \\
\text { pleta }\end{array}$ & 100 & 22 & \\
\hline Universitaria completa & 63 & 17 & \\
\hline Posgrado & 19 & 2 & \\
\hline Total & 411 & 109 & \\
\hline
\end{tabular}

Nota. ${ }^{*} \mathrm{p}<0.05 ; * * \mathrm{p}<0.01$.

Fuente: elaboración propia. 
Finalmente, para clasificar los factores de riesgo como los protectores de ansiedad estado, se revisó la significancia, el peso predictivo y los odds ratio del modelo. Este análisis evidenció que la depresión $(\mathrm{OR}=1.296 \mathrm{IC}=1.218$ - 1.378), se constituye como factor de riesgo para ansiedad estado, mientras que la felicidad $(\mathrm{OR}=0.493 \mathrm{IC}=0.348-0.699)$ es un factor protector (Tabla 4).

Concordantemente, el estadístico Wald indica que tanto la depresión como la felicidad son las variables con mayor peso predictivo. Es decir, ambas variables explican en mayor cantidad la variabilidad del modelo. De esta forma y al llevar el estadístico
Wald a porcentajes, se observa que la depresión explica un $60 \%$ de ansiedad estado (entendida como factor de riesgo), mientras que la felicidad es capaz de explicar un $14 \%$ del atributo (entendida como factor protector) (Tabla 4).

\section{Modelo predictivo para la ansiedad rasgo}

La evaluación de la capacidad predictiva de las variables de estudio en relación con la ansiedad rasgo, se realizó, al igual que en el caso anterior, por medio de una regresión logística (Tabla 5), probándose un modelo con las variables psicológicas anteriormente

TABLA 4

Regresión logística para la predicción de la ansiedad estado

\begin{tabular}{|c|c|c|c|c|c|c|c|}
\hline & $\mathrm{B}$ & E.T. & Wald & Sig. & OR & \multicolumn{2}{|c|}{ I.C. $95 \%$ para OR } \\
\hline Edad & 0.049 & 0.065 & 0.572 & 0.449 & 1.05 & 0.925 & 1.192 \\
\hline Sexo & 0.146 & 0.295 & 0.245 & 0.621 & 1.157 & 0.649 & 2.061 \\
\hline Estado civil & 20.729 & 10541.166 & 0 & 0.998 & $1.006 \mathrm{E} 9$ & 0 & \\
\hline N. Educacional & & & 7.76 & 0.256 & & & \\
\hline Básico incompleto & -2.689 & 1.653 & 2.644 & 0.104 & 0.068 & 0.003 & 1.737 \\
\hline Media incompleta & -2.12 & 1.586 & 1.785 & 0.181 & 0.12 & 0.005 & 2.69 \\
\hline Media completa & -2.401 & 1.573 & 2.329 & 0.127 & 0.091 & 0.004 & 1.979 \\
\hline Univ. incompleta & -1.56 & 1.404 & 1.234 & 0.267 & 0.21 & 0.013 & 3.294 \\
\hline Univ. completa & -0.892 & 1.225 & 0.531 & 0.466 & 0.41 & 0.037 & 4.518 \\
\hline Post grado & -1.697 & 0.904 & 3.525 & 0.06 & 0.183 & 0.031 & 1.077 \\
\hline Ocupación & & & 1.113 & 0.953 & & & \\
\hline Trabajos menores & -0.217 & 1.524 & 0.020 & 0.887 & 0.805 & 0.041 & 15.973 \\
\hline Oficio menor & -0.276 & 1.518 & 0.033 & 0.856 & 0.759 & 0.039 & 14.876 \\
\hline Obrero calificado & 0.015 & 1.324 & 0.000 & 0.991 & 1.015 & 0.076 & 13.592 \\
\hline Empl. Administrativ. & -0.434 & 1.297 & 0.112 & 0.738 & 0.648 & 0.051 & 8.239 \\
\hline Ejecutivo medio & -0.143 & 1.056 & 0.018 & 0.892 & 0.867 & 0.109 & 6.864 \\
\hline N. socioeconómico & & & 4.324 & 0.504 & & & \\
\hline Bajo & 1.308 & 2.519 & 0.270 & 0.604 & 3.698 & 0.027 & 515.699 \\
\hline Medio bajo & 0.382 & 2.412 & 0.025 & 0.874 & 1.465 & 0.013 & 165.683 \\
\hline Medio & 0.187 & 2.235 & 0.007 & 0.933 & 1.205 & 0.015 & 96.302 \\
\hline Medio alto & 0.022 & 1.893 & 0.000 & 0.991 & 1.022 & 0.025 & 41.762 \\
\hline Alto & 0.838 & 1.271 & 0.435 & 0.51 & 2.311 & 0.192 & 27.889 \\
\hline Depresión & 0.259 & 0.031 & 67.982 & $0 * *$ & 1.296 & 1.218 & 1.378 \\
\hline Optimismo & -0.075 & 0.048 & 2.392 & 0.122 & 0.928 & 0.844 & 1.02 \\
\hline Felicidad & -0.707 & 0.178 & 15.743 & $0 * *$ & 0.493 & 0.348 & 0.699 \\
\hline Constante & -19.35 & 10541.166 & 0 & 0.999 & 0 & & \\
\hline
\end{tabular}

Nota. $* *$ p $<$ 0.01. Variable(s) introducida(s): edad, sexo, estado civil, nivel educacional, ocupación, nivel socioeconómico, depresión, optimismo, felicidad.

Fuente: elaboración propia. 
descritas. El modelo resultante fue adecuado para la prueba de Hosmer y Lemeshow ( $p=0.975)$, indicando que no hay diferencias estadísticamente significativas entre las clasificaciones observadas y las predichas.

Por su parte el estadístico $R^{2}$ de Nagelkerke estima un valor de ajuste adecuado (0.648), lo que lleva a aceptar formalmente un buen ajuste del modelo, estableciendo la existencia de una correlación fuerte $(r=0.8)$ entre todas las variables predictoras y la ansiedad rasgo.

Asimismo, el modelo resultante evidenció una adecuada especificidad (95.6\%) y sensibilidad
(67\%), es decir, que clasifica correctamente a aquellas personas que efectivamente no presentan la ansiedad rasgo, como a aquellas personas que sí tienen el atributo. Igualmente, se observa un adecuado potencial global (89.6\%), es decir que existe buena capacidad predictiva global del modelo.

Finalmente, y al igual que en el modelo anterior, para clasificar los factores de riesgo como los protectores de ansiedad rasgo, se revisó la significancia, el peso predictivo y los odds ratio del modelo. Este análisis evidenció que la depresión $(\mathrm{OR}=1.355$ $\mathrm{IC}=1.258-1.459)$, se constituye como factor de

TABLA 5

Regresión logística para la predicción de la ansiedad rasgo

\begin{tabular}{lccccccc}
\hline & B & E.T. & Wald & Sig. & OR & \multicolumn{2}{c}{ I.C. 95\% para OR } \\
\hline Edad & -0.005 & 0.076 & 0.004 & 0.948 & 0.995 & 0.857 & 1.155 \\
Sexo & 0.469 & 0.355 & 1.742 & 0.187 & 1.598 & 0.797 & 3.207 \\
Estado civil & 0.449 & 1.432 & 0.098 & 0.754 & 1.567 & 0.095 & 25.937 \\
N. educacional & & & 5.165 & 0.523 & & & \\
Básica incompleta & -1.776 & 2.128 & 0.697 & 0.404 & 0.169 & 0.003 & 10.962 \\
Media incompleta & -1.595 & 2.087 & 0.584 & 0.445 & 0.203 & 0.003 & 12.122 \\
Media completa & -2.336 & 2.048 & 1.301 & 0.254 & 0.097 & 0.002 & 5.355 \\
Univ. incompleta & -0.75 & 1.867 & 0.161 & 0.688 & 0.472 & 0.012 & 18.362 \\
Univ. completa & -0.836 & 1.641 & 0.259 & 0.610 & 0.433 & 0.017 & 10.813 \\
Postgrado & -0.109 & 1.236 & 0.008 & 0.930 & 0.897 & 0.08 & 10.113 \\
Ocupación & & & 1.909 & 0.862 & & & \\
Trabajos menores & -2.31 & 2.178 & 1.125 & 0.289 & 0.099 & 0.001 & 7.093 \\
Oficio menor & -1.668 & 2.158 & 0.598 & 0.439 & 0.189 & 0.003 & 12.944 \\
Obrero calificado & -1.855 & 1.946 & 0.909 & 0.340 & 0.156 & 0.003 & 7.089 \\
Empl. Administrat. & -1.850 & 1.888 & 0.961 & 0.327 & 0.157 & 0.004 & 6.358 \\
Ejecutivo medio & -1.144 & 1.537 & 0.554 & 0.457 & 0.318 & 0.016 & 6.48 \\
N. socioeconómico & & & 8.084 & 0.152 & & & \\
Bajo & 5.07 & 3.402 & 2.221 & 0.136 & 159.231 & 0.202 & 125243 \\
Medio bajo & 3.302 & 3.249 & 1.032 & 0.310 & 27.157 & 0.047 & 15839.76 \\
Medio & 4.315 & 3.042 & 2.013 & 0.156 & 74.817 & 0.193 & 29046.12 \\
Medio alto & 3.052 & 2.56 & 1.421 & 0.233 & 21.158 & 0.14 & 3196.667 \\
Alto & 2.773 & 1.874 & 2.189 & 0.139 & 16.009 & 0.406 & 630.825 \\
Depresión & 0.304 & 0.038 & 64.272 & $0 * *$ & 1.355 & 1.258 & 1.459 \\
Optimismo & -0.263 & 0.061 & 18.383 & $0 * *$ & 0.769 & 0.682 & 0.867 \\
Felicidad & -0.747 & 0.198 & 14.205 & $0 * *$ & 0.474 & 0.322 & 0.699 \\
Constante & 1.513 & 3.035 & 0.249 & 0.618 & 4.542 & & \\
\hline Nota $<0.01$. & & & & & & & \\
& & & & & & &
\end{tabular}

Nota. $* * p<0.01$. Variable(s) introducida(s): edad. sexo. estado civil. nivel educacional. ocupación. nivel socioeconómico. depresión. optimismo. felicidad.

Fuente: elaboración propia. 
riesgo para la ansiedad rasgo, mientras que la felicidad $(\mathrm{OR}=0.474 \mathrm{IC}=0.322-0.699)$ y el optimismo $(\mathrm{OR}=0.769 \mathrm{IC}=0.682-0.867)$ serían factores protectores (Tabla 5).

Coherentemente, el estadístico Wald indica que tanto la depresión como la felicidad y el optimismo son las variables con mayor peso predictivo. Es decir, estas tres variables explican en mayor cantidad la variabilidad del modelo. De esta forma y al llevar el estadístico Wald a porcentajes, se observa que la depresión explica un 49 \% de la ansiedad rasgo (entendida como factor de riesgo), y que la felicidad es capaz de explicar un $11 \%$, mientras que el optimismo explica un $14 \%$ (ambas variables como factor protector) (Tabla 5).

\section{Discusión}

Se analizaron en conjunto las posibles variables protectoras y de riesgo para la ansiedad. En relación con las variables sociodemográficas, los resultados para la edad, si bien son similares a estudios que indican que esta variable de riesgo se encuentra presente en mayor medida en las personas jóvenes, la presente investigación difiere al indicar que más bien son los sujetos entre los 18 y los 24 años de edad los que presentan mayor asociación con esta variable. Esto es específicamente discordante con la Encuesta Nacional de Salud (2003) que informa que en Chile son las personas entre los 25 y los 44 años de edad las que presentan mayor prevalencia de sintomatología en salud mental.

En cuanto al sexo, las mujeres presentaron mayor presencia de ansiedad rasgo que los hombres, dato que es coincidente con trabajos anteriores en los que se ha identificado una mayor tendencia a la ansiedad por parte de las mujeres (Almedilla-Zafra, Ortega-Toro, 2009; Almedilla-Zafra, Gum, KingKallimanis \& Kohn, 2009; Micin \& Bagladi, 211; Ortega-Toro \& Madrid-Garrido, 2008; Pimentel \& Cova, 2011). Igualmente, estos resultados han sido corroborados por estudios de prevalencia en Chile (Vicente et al., 2002).

Respecto de la capacidad predictiva para la ansiedad, los resultados indican que solo la depresión puede ser considerada como un factor de riesgo para la ansiedad. Lo que es coherente con diversas investigaciones y modelos teóricos que muestran una considerable relación y comorbilidad entre estas variables (Clark \& Watson, 1991; Forand et al., 2010; Páez, Jofré, Azpiroz \& De Bartoli, 2009; Watson et al., 1998).

Respecto de los factores protectores para esta sintomatología, el modelo evidencia para ambos tipos de ansiedades que la variable felicidad se constituyó como un factor de este tipo, con adecuada significancia estadística, lo que es concordante con estudios recientes (Fredrickson \& Losada, 2005; Fredrickson \& Tugade, 2003; Lyubomirsky, 2008).

En este mismo sentido, los resultados indican que el optimismo sería un factor protector para la ansiedad, lo que es coincidente con estudios que aunque no analizaron el optimismo como factor de riesgo o protector si han encontrado relaciones inversas con variables negativas como la ansiedad (Abdel-Khalek, 2006; Brydon et al., 2009; Chico \& Ferrando, 2008; De Moor et al., 2006; Kivimaki et al., 2005; Lobel et al., 2000; Puskar et al., 2008; Vera, 2006; Vera-Villarroel et al., 2009a; VeraVillarroel et al., 2010), lo cual puede indicar no solo que exista una relación inversa con estados psicológicos negativos, sino tal como lo plantean los resultados del presente estudio, sería más bien un factor que pudiera estar protegiendo a las personas de experimentar mayor ansiedad.

A pesar de estos resultados, la presente investigación exhibe algunas limitaciones que deben tenerse presentes; una de ellas es que la muestra fue no probabilística, por lo que los datos obtenidos deben ser considerados con cautela, pues no es posible su generalización, ni el cálculo preciso del error estándar necesario para realizar extrapolaciones adecuadas (Hernández, Fernández \& Baptista, 1991),

El presente estudio sugiere algunas proyecciones para futuras investigaciones como la incorporación y control de otras variables sociodemográficas que puedan estar actuando como variables intervinientes o protectoras y de riesgo, como por ejemplo el ejercicio físico, aspectos culturales, número de 
hijos, etc., así como la comparación con otro tipo de trastornos psicológicos, controlando el efecto de variables comórbidas. Igualmente, sería esperable la confirmación o no de los datos obtenidos en este estudio a partir de investigaciones que desarrollen un modelo causal, donde la bondad de ajuste entre lo observado y la teoría permita explicar la linealidad de los datos.

Finalmente, se presentan los modelos encontrados a partir del presente trabajo, donde se combinan las variables positivas, negativas y sociodemográficas de estudio (Figuras 1 y 2).

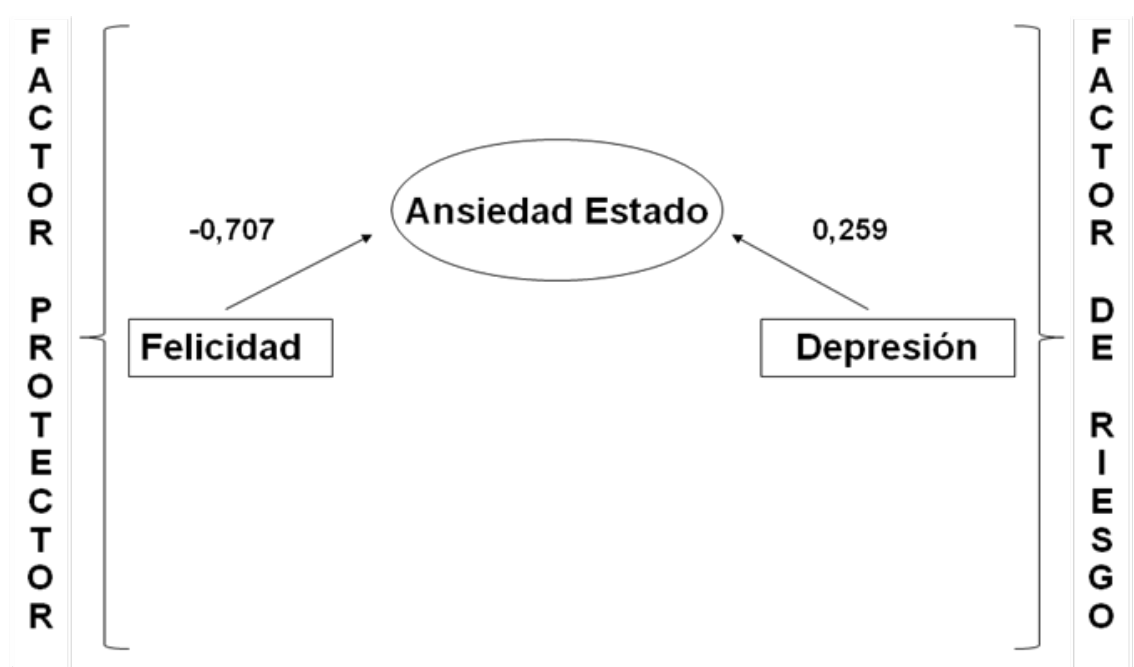

Figura 1. Modelo predictivo de la Ansiedad Estado.

Fuente: elaboración propia.

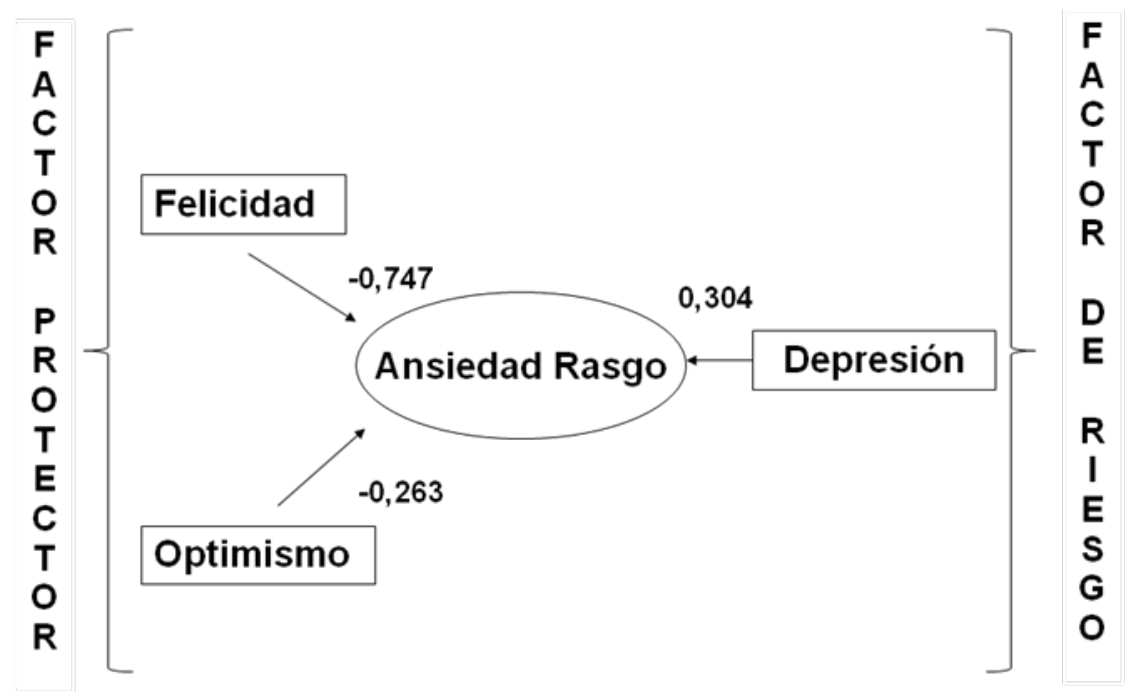

Figura 2. Modelo predictivo de la Ansiedad Rasgo.

Fuente: elaboración propia 


\section{Referencias}

Abdel-Khalek, A. (2006). Measuring happiness with a single-item scale. Social Behavior and Personality, $34,139-150$.

Adimark. (2000). El Nivel Socio Económico Esomar. Manual de Aplicación. Extraído el 1 de septiembre, 2010. de http://www.microweb.cl/idm/documentos/ESOMAR.pdf

Almedilla-Zafra, A. \& Ortega-Toro, E. (2009). Incidencia de la práctica de actividad física sobre la ansiedad y depresión en mujeres: perfiles de riesgo. Universitas Psychologica, 8(1), 105-116.

Almedilla-Zafra, A., Ortega-Toro, E. \& Madrid-Garrido, J. (2009). Variables sociodemográficas, ejercicio físico, ansiedad y depresión en mujeres: un estudio correlacional. Revista Internacional de Medicina y Ciencias de la Actividad Física y el Deporte, 31. 224-243.

Arcas, S. \& Cano, A. (1999). Procesos cognitivos en el trastorno de ansiedad generalizada, según el paradigma del procesamiento de la información. Recuperado el 19 de diciembre, 2010. de http://www.psiquiatria. com/psicologia/vol3num1/art_6.htm

Beck, A., Rush, A., Shaw, B. \& Emery, G. (1983). Terapia cognitiva de la depresión. Bilbao: Descleé de Broker.

Beutel, M.E., Bleichner, F., von Heymann, F., Tritt, K., \& Hardt, J. (2011). Inpatient psychosomatic treatment of anxiety disorders: Comorbidities, predictors, and outcomes. Intenational Journal of Clinical and Health Psychology, 11, 443-457.

Brydon, L., Walker, C., Wawrzyniak, A. J., Chart, H. \& Syeptoc, A. (2009). Dispositional optimism and stress-induced changes in immunity and negative mood. Behavioral and Inmunity, 6, 810-816.

Contreras, F. \& Esguerra, G. (2006). Psicología positiva: una nueva perspectiva en Psicología. Diversitas: Perspectivas en Psicología, 3, 311-319.

Chico, E. \& Ferrando, P. (2008). Variables cognitivas y afectivas como predictoras de satisfacción en la vida. Psicothema, 20. 408-412.

Clark, L. \& Watson, D. (1991). Tripartite model of anxiety and depression: Psychometric evidence and taxonomic implications. Journal of Abnormal Psychology, 100. 316-336.
Cuadra-Peralta, A., Veloso-Besio, C., Ibergaray, M., \& Rocha, M. (2010). Resultados de la psicoterapia positiva en pacientes con depresión. Terapia Psicológica, 28, 127-134.

Cova, F., Rincón, P., \& Mellipán, R. (2011). Evaluación de la eficacia de un programa preventivo para la depresión en adolescentes de sexo femenino. Terapia Psicológica, 29, 245-250.

De Moor, J. S., De Moor, C. A., Basen-Engquist, K., Kudelka, A., Bevers, M. W. \& Cohen, L. (2006). Optimism, distress, health-related quality of life, and change in cancer antigen 125 among patients with ovarian cancer undergoing chemotherapy. Psychosomatic Medicine, 4, 555-562.

Encuesta Nacional de Salud (2003). Resultados de la I Encuesta de Salud Chile 2003. Ministerio de Salud. Forand, N., Gunthert, K., Cohen, L., Butler, A., \& Beck, J. (2010). Preliminary evidence that anxiety is associated with accelerated response in cognitive therapy for depression. Cognitive Therapy and Research, 35(2), 151-160. Extraído el 1 de marzo, 2011. de http://www.springerlink.com/content/ t885h31734738266/

Fredrickson B. L. (1998). What good are positive emotions? Review of General Psychology, 2, 300-319.

Fredrickson, B. L. \& Losada, M. F. (2005). Positive affect and the complex dynamics of human flourishing. American Psychologist, 60. 678-686.

Fredrickson, B. L. \& Tugade, M. M. (2003). What good are positive emotions in crises? A prospective study of resilience and emotions following the terrorist attacks on the USA on September 11 ${ }^{\text {th }}, 2001$. Journal of Personality and Social Psychology, 84, 365-376.

Gable, S. \& Haidt, J. (2005). What (and Why) is positive psychology? Review of General Psychology, 9, 103-110.

Gum, A. M., King-Kallimanis, B. \& Kohn, R. (2009). Prevalence of mood, anxiety, and substance-abuse disorder for older Americans in the national comorbidity survey-replication. American Journal of Geriatric Psychiatry, 17, 769-781.

Hart, S., Vella, L. \& Mohr, D. (2008). Relationships among depressive symptoms, benefit-finding, optimism, and positive affect in multiple sclerosis patients after psychotherapy for depression. Health Psychology, 27(2), 230-238. 
Hernández, R., Fernández, C. \& Baptista, P. (1991). Metodología de la investigación. México: McGrawHill.

Kessler, R. C. \& Üstün, T. B. (2004). The World Mental Health (WMH) Survey Initiative version of the World Health Organization (WHO) Composite International Diagnostic Interview (CIDI). International Journal of Methods in Psychiatric Research, 13, 93-121.

Kivimaki, M., Elovainio, M., Singh-Manoux, A., Vahtera, J., Helenius, H. \& Pentti, J. (2005). Optimism and pessimism as predictors of change in health after death or onset of severe illness in family. Health Psychology, 24, 413-421.

Koopmans, T. A., Geleijnse, J. M., Zitman, F. G. \& Giltay, E. J. (2010). Effects of happiness on allcause mortality during 15 years of follow-up: The Arnhen Elderly Study. Journal of Happiness Studies, 11(1), 113-124.

Laranjeira, C. A. (2008). Tradução e validação portuguesa do Revised Life Orientation Test (LOT-R). Universitas Psychologica, 7(2), 469-476.

Lobel, M., DeVincent, C., Kaminer, A. \& Meyer, B. (2000). The impact of prenatal maternal stress and optimistic disposition on birth outcomes in medically high-risk women. Health Psychology, 19, 544-553.

Lyubomirsky, S. (2008). La ciencia de la felicidad. Barcelona: Uranos.

Lyubomirsky, S. \& Lepper, H. (1999). A measure of subjective hapiness: Preliminary realiability and construct validation. Social Indicators Research, 46, 137-155.

Martínez, M. (2006). El estudio científico de las fortalezas trascendentales desde la psicología positiva. Clínica y Salud, 17, 245-258.

Melipillán Araneda, R., Cova Solar, F., Rincón González, P. \& Valdivia Peralta, M. (2008). Propiedades psicométricas del Inventario de Depresión de Beck-II en adolescentes chilenos. Terapia Psicológica, 26, 59-69.

Micin, S., \& Bagladi, V. (2011). Salud mental en estudiantes universitarios: Incidencia de psicopatología y antecedentes de conducta suicida en población que acude a un servicio de salud estudiantil. Terapia Psicológica, 29, 53-64.
Moyano, D. E., Flores, M.E., \& Soromaa, H. (2011). Fiabilidad y validez de constructo del test munsh para medir felicidad, en población de adultos mayores chilenos. Universitas Psychologica, 10, 567-580.

Moyano, E. \& Ramos, N. (2007). Bienestar subjetivo: midiendo satisfacción vital, felicidad y salud en población chilena de la Región del Maule. Universum, 22, 177-193.

Mustaca, A.E., Kamenetzky, G., \& Vera-Villarroel, P. (2010). Relaciones entre variables positivas y negativas en una muestra de estudiantes argentinos. Revista Argentina de Clínica y Psicología, 19, 227-235.

Otero-López, J. M., Luengo, A., Romero, E., Gómez, J. A. \& Castro, C. (1998). Psicología de personalidad. Manual de prácticas. Barcelona: Ariel Practicum.

Páez, A., Jofré, M., Azpiroz, C. \& De Bartoli, M. A. (2009). Ansiedad y depresión en pacientes con insuficiencia renal crónica en tratamiento de diálisis. Universitas Psychologica, 8(1), 117-124.

Pimentel, M., \& Cova, F. (2011). Efectos de la rumiación y la preocupación en el desarrollo de sintomatología depresiva y ansiosa en estudiantes universitarios de la ciudad de concepción, Chile. Terapia Psicológica, 29, 43-52.

Piqueras, J.A., Kuhne, W., Vera-Villarroel, P., van Straten, A., \& Cuijpers, P. (2011) Happiness and health behaviours in chilean college students: A cross-sectional survey. BMC Public Health, 11, 443. doi:10.1186/1471-2458-11-443.

Puskar, K., Ren, D., Bernardo, L. M., Haley, T. \& Stark, K. H. (2008). Anger correlated with psychosocial variables in rural youth. Issues in Comprehensive Pediatric Nursing, 3, 71-87.

Retana-Franco, B., \& Sánchez-Aragón, R. (2010). Rastreando en el pasado formas de regular la felicidad, la tristeza, el amor, el enojo y el miedo. UniversitasPsychologica, 9, 179-197.

Salkind, N. (1998). Métodos de investigación. México: Pretince Hall.

Sandín, B. (1995). Teorías sobre los trastornos de ansiedad. En A. Bellooch, B. Sandín \& F. Ramos (Eds.), Manual de psicopatología (pp. 113-169). Madrid: McGraw-Hill.

Sanz, J. \& Vázquez, C. (1998). Fiabilidad, validez y datos normativos del Inventario para la Depresión de Beck. Psicothema, 10. 303-318. 
Scheier, M. F., Carver, C. \& Bridges, M. W. (1994). Distinguishing optimism from neuroticism (and trait anxiety, self-mastery, and self-esteem): A reevaluation of the Life Orientation Test. Journal of Personality and Social Psychology, 67, 1063-1078.

Seligman, M. E. P., Steen, T. A., Park, N. \& Peterson, C. (2005). Positive psychology progress empirical validation of interventions. American Psychologist, 60. 410-421.

Spielberger, Ch., Gorsuch, R. \& Luschene, R. (1970). Manual for the State-Trait Anxiety Inventory. Palo Alto, CA: Consulting Psychologist Press [Adaptación española, TEA, 1982].

Taylor, S., Kemeny, M., Reed, G., Bower, J. \& Gruenewald, T. (2000). Psychological resources, positive illusions, and health. American Psychologist, 55, 99-109.

Vázquez, C., Hervás, G., \& Ho, S. (2006). Intervenciones clínicas basadas en la psicología positiva: fundamentos y aplicaciones. Psicología Conductual, 14, 401-432.

Vázquez, C., Hérvas, G., Rahona, J. J. \& Gómez, D. (2009). Bienestar psicológico y salud: aportaciones desde la psicología positiva. Annuary of Clinical and Health Psychology, 5, 15-28.

Vázquez, C. \& Sanz, J. (1997). Fiabilidad y valores normativos de la versión española del Inventario para la Depresión de Beck de 1978. Clínica y Salud, 8(3), 403-422.

Vera, B. (2006). Psicología positiva: una nueva forma de entender la Psicología. Papeles del Psicólogo, 27, 3-8.

Vera-Villarroel, P.E. \& Buela-Casal, G. (2000). Relaciones entre ansiedad y estilo atributivo. Revista Mexicana de Psicología, 17, 137-142.

Vera-Villarroel, P., Buela-Casal, B., Celis-Atenas, K., Córdova-Rubio, N., Encina-Olea, N.\& Spielberger, CD (2008). Chilean experimental version of the State-Trait Depression Questionnaire (ST-DEP): Trait sub-scale (T-DEP). International Journal of Clinical and Health Psychology, 8, 563-575.

Vera-Villarroel, P., Celis-Atenas, K. \& Córdova-Rubio, N. (2011). Evaluación de la Felicidad: análisis psicométrico de la Escala de Felicidad Subjetiva en población chilena. Terapia Psicológica, 29, 127-133.
Vera-Villarroel, P., Celis-Atenas, K., Córdova-Rubio, N., Buela-Casal, G. \& Spielberger, C. D. (2007). Preliminary analysis and normative data of the State-Trait Anxiety Inventory (STAI) in adolescent and adults of Santiago, Chile. Terapia Psicológica, 25, 155-162.

Vera-Villarroel, P., Córdova-Rubio, N. \& Celis-Atenas, K. (2009a). Optimismo versus Autoestima: implicancia para la psicología clínica y la psicoterapia. Revista Argentina de Clínica Psicológica, 18, 21-30.

Vera-Villarroel, P., Córdova-Rubio, N. \& Celis-Atenas, K. (2009b). Evaluación del optimismo: un análisis preliminar del Life Orientation Test versión revisada (LOT-R) en población chilena. Universitas Psychologica, 8(1), 61-68.

Vera-Villarroel, P., Zych, I., Celis-Atenas, K., CórdovaRubio, N., \& Buela-Casal, G. (2011). Chilean Validation of The Posttraumatic Stress Disorder Checklist - Civilian Version (Pcl-C) After The Earthquake On February 27, 2010. Psychological Reports. 109, 47-58.

Vicente, B., Rioseco, P., Saldivia, S., Kohn, R. \& Torres, S. (2002). Estudio chileno de prevalencia de patología psiquiátrica. Revista Médica de Chile, 130. 527-536.

Vittorio, G., Fagnani, C., Alessandri, G., Steca, P., Gigantesco, A., Cavalli, L., et al. (2009). Human optimal functioning: The genetics of positive orientation towards self, life, and the future. Behavior Genetics, 39, 277-284.

Watson, D., Clark, L. A. \& Carey, G. (1998). Positive and negative affectivity and their relation to anxiety and depressive disorders. Journal of Abnormal Psychology, 97, 346-353.

Watson, D., Clark, L.A., \& Stasik, S.M. (2011). Emotions and the emotional disorders: A quantitative hierarchical perspective. International Journal of Clinical and Health Psychology, 11, 429-442.

Wolitzky-Taylor, K. B., Castriotta, N., Lenze, E. J., Stanley, M. A. \& Craske, M. G. (2010). Anxiety disorders in older adults: A comprensive review. Depression Anxiety, 27, 190-211. 